Meta

Journal des traducteurs

Translators' Journal

\title{
L'ordinateur au service du terminologue : maître ou esclave
}

\section{J. Albert Bachrach}

Volume 16, numéro 1-2, mars 1971

Actes du colloque international de linguistique et de traduction.

Montréal, 30 septembre - 3 octobre 1970

URI : https://id.erudit.org/iderudit/001939ar

DOI : https://doi.org/10.7202/001939ar

Aller au sommaire du numéro

Éditeur(s)

Les Presses de l'Université de Montréal

ISSN

0026-0452 (imprimé)

1492-1421 (numérique)

Découvrir la revue

Citer cet article

Bachrach, J. A. (1971). L'ordinateur au service du terminologue : maître ou esclave. Meta, 16(1-2), 105-115. https://doi.org/10.7202/001939ar d'utilisation que vous pouvez consulter en ligne.

https://apropos.erudit.org/fr/usagers/politique-dutilisation/ 


\section{L'ordinateur au service du terminologue: maître ou esclave}

À notre époque, nous sommes tellement habitués à nous servir de divers appareils et machines pour l'accomplissement de maintes tâches pénibles ou fastidieuses que nous oublions parfois les servitudes visibles et invisibles que cela comporte. Les avantages qu'ils offrent ne sont réels que dans la mesure où ils marchent sans défaillir. Les automates nous permettent d'accomplir plus dans un laps de temps donné, mais ils contribuent aussi à l'accélération du rythme de la vie, ce qui nous oblige à nous spécialiser toujours davantage. Si paradoxal que cela puisse paraître, toutes ces extensions de nos moyens d'action dont parle MacLuhan ${ }^{1}$, ne semblent pas avoir contribué à la diffusion du savoir universel, mais plutôt à la formation d'experts confortablement installés dans leur propre champ d'activité. Ils font usage des machines, mais ils ne se soucient en général que fort peu des détails de leur fonctionnement. Et tout va très bien pour autant qu'elles continuent à marcher sans faiblesse. Lorsqu'une panne mécanique intervient, toute l'activité s'arrête en attendant la venue de cet autre spécialiste qu'est le technicien. Mais, attendre le technicien équivaut souvent à attendre Godot !

Lorsque nous parlons des machines électroniques qui aident l'homme à résoudre des problèmes, nous sommes tentés de penser uniquement aux problèmes numériques très compliqués. Est-ce parce qu'on a été trop longtemps sceptique à cet égard, que l'on a tendance à oublier que l'ordinateur intervient de plus en plus dans la solution des problèmes non numériques ?

Dans cet article, nous traiterons du profit que l'on peut tirer de l'emploi de l'ordinateur dans l'élaboration d'un système de consultation automatique de dictionnaires, le système DICAUTOM, élaboré en collaboration avec le Centre de linguistique automatique appliquée de l'Université libre de Bruxelles ${ }^{2}$.

\section{ANALYSE DU PROBLÈME}

Le préalable de toute utilisation d'un ordinateur est qu'il faut bien se rendre compte qu'il ne s'agit point d'une machine qui peut agir seule, mais bien au contraire d'un instrument qui ne peut donner satisfaction qu'à la condition que l'homme lui fournisse des instructions claires et précises.

1. Marshall MacLuhan, Understanding Media.

2. DICAUTOM (consultation automatique de dictionnaires pour traducteurs humains), la Traduction automatique, IV, 3 (septembre 1963). 
Avant de commencer la mise au point d'un système de traitement de l'information où intervient l'ordinateur, il faut que le problème soit analysé à fond et que l'initiateur du projet ait bien clairement à l'esprit le résultat final qu'il veut obtenir. Il ne doit pas non plus perdre de vue que son système doit servir un but pratique et que le résultat produit par l'ordinateur doit répondre aux besoins du client. Pour réaliser tout cela, il importe bien sûr de tenir compte des possibilités qu'offre l'ordinateur auquel on a accès, mais aussi de ne pas oublier de penser, autant que possible, aux améliorations qui pourraient être obtenues en cas d'extension de la capacité de la machine. Il faut à tout prix éviter que l'imprimante produise des montagnes de papier qui nécessitent l'étude de codes multiples avant que l'utilisateur puisse y trouver l'information recherchée. Malheureusement, des systèmes de ce genre existent et c'est à cause d'eux que d'autres initiatives mieux structurées ont été souvent discréditées.

Afin de pouvoir satisfaire le client, il convient d'analyser ses besoins, d'étudier le déroulement de son travail ct les outils de travail dont il dispose, de connaître le degré de précision et la rapidité d'accès souhaités pour les informations à fournir.

Dans le cas qui nous occupe, il faut venir en aide au traducteur qui consacre approximativement $50 \%$ de son temps à des recherches terminologiques souvent vaines. Ce nouvel instrument de travail est donc avant tout destiné à diminuer la durée de ces recherches. Parmi les outils dont dispose le traducteur, les dictionnaires et glossaires occupent une place importante. Malgré les désavantages qu'ils présentent, contre lesquels Jean Maillot ${ }^{3}$ et bien d'autres mettent"en garde à juste titre, ces ouvrages représentent des sources d'information auxquelles les traducteurs ont le plus facilement accès.

L'accélération de l'évolution du progrès scientifique et technique est cependant telle que ces ouvrages ne sauront guère contenir tous les néologismes dont fourmillent les descriptions des nouveaux procédés techniques ou des applications des dernières découvertes scientifiques. Par les moyens traditionnels, il sera pratiquement impossible de repérer, d'enregistrer et de publier tous ces termes nouveaux, souvent inventés de toutes pièces par les auteurs de ces textes. L'ordinateur permettant l'enregistrement des données et leur mise à jour continuelle, ainsi que la recherche ultra-rapide, il semblerait facile de simplement emmagasiner en mémoire électronique le contenu de tous les dictionnaires et glossaires dont on dispose et de prévoir leur mise à jour. Mais, cela n'est pas très recommandable parce qu'ils sont élaborés suivant des principes parfois très différents.

\section{ÉLABORATION D'UN FICHIER PHRASÉOLOGIQUE}

Il $\mathrm{y}$ a, toutefois, un point que tous ces ouvrages ont en commun, notamment qu'au départ leur contenu se présente sous forme d'un fichier. Or, c'est justement la constitution d'un tel fichier qui formera le point de départ de nos travaux également.

L'utilisateur principal du système sera le traducteúr, qui est appelé à rendre dans une langue donnée des textes rédigés dans une autre langue; notre fichier sera donc plurilingue. Il ne faut pas oublier que le traducteur qui est au service d'une grande organisation internationale n'a pratiquement pas la possibilité de se

3. Jean Maillot, la Traduction scientifique et technique, Paris, Eyrolles, 1970. 
spécialiser, le champ d'action étant trop vaste. Le DICAUTOM doit done englober presque tous les domaines de l'activité humaine. Il est évident que, même avec un effectif important, il sera absolument impossible de commencer tout à la fois. Il faut procéder par petits secteurs, un peu comme le système des jeux de construction, tel le meccano.

Afin d'éviter que les erreurs de traduction qui ont pu échapper à l'attention du réviseur soient «entrées » dans le système, l'analyse se fera à partir de textes originaux pour chacune des langues de travail. Partant de la prémisse que, d'une part, le mot et même le terme technique ou scientifique ne détient sa signification particulière que du contexte dans lequel il figure et que, d'autre part, le traducteur a souvent moins de mal à trouver le terme exact recherché que les éléments de la phrase qu'il faut $y$ assortir, le fichier ne contiendra que des termes dans un contexte.

Cette option est aussi fondée sur le fait que dans beaucoup de langues, nombre de mots composés se présentent comme un groupe dont les éléments sont séparés par des blancs. Il serait par exemple difficile de définir le sens exact du terme français « pont » s'il n'est pas précédé par le verbe «faire» ce qui donne «faire le pont» ou suivi d'un adjectif tel «roulant», ce qui donne «pont roulant» (overhead travelling crane), sans oublier le «pont thermique » (cold bridge) qui est encore tout autre chose.

Une fois que le domaine, dont on veut recenser le vocabulaire, est bien délimité, une documentation est rassemblée pour chacune des langues de travail. Pour nous, ce sont les quatre langues officielles des communautés européennes : l'allemand, le français, l'italien et le néerlandais, et en plus l'autre grande langue de l'Occident : l'anglais. Chaque terminologue procède d'abord à l'analyse des documents rédigés dans sa propre langue.

Ensuite, il passe les textes et le fichier phraséologique unilingue, qui en résulte, à un de ses collègues qui est responsable d'une autre langue et, qui lui, à son tour, à l'aide de la documentation originale dont il dispose cherche les équivalents dans sa langue. La ronde continue ainsi jusqu'à ce que toutes les phrases soient reproduites dans toutes les langues voulues. Tous les cas douteux ou ambigus sont soumis aux experts avec lesquels le service est en rapport.

Les fiches contiennent outre le numéro chronologique de la phrase, une indication du code-matière, les références bibliographiques et une définition, chaque fois que nous jugeons que le contexte ne contient pas suffisamment d'informations au sujet de la notion à laquelle le terme se réfère. Le travail d'analyse terminé pour toutes les langues, on dispose d'un fichier phraséologique plurilingue dactylographié. Tant que la lecture optique ne sera pas plus perfectionnée, le contenu du fichier devra ensuite être entièrement transcrit sur cartes perforées. C'est à ce stade qu'apparaît la première servitude qui est d'ailleurs peut-être la plus tracassante pour le terminologue.

\section{CONSULTATION AUTOMATIQUE}

EN VUE DE LA PUBLICATION DE GLOSSAIRES

Lors de la conception du DICAUTOM nous avons jugé qu'il fallait limiter à 180 positions la longueur de la locution à insérer. Nous avons craint de perdre 
un trop grand nombre de positions pour les accents, les majuscules et autres signes. Il faut dire qu'à l'époque nous disposions d'un ordinateur IBM 1401 et que le système a été conçu pour la réalisation de deux objectifs bien distincts, à savoir la publication de glossaires et la consultation automatique de la mémoire. Une trop grande longueur de phrase peut gêner une bonne mise en page et diminuer l'intelligibilité. Le terminologue doit donc être constamment vigilant pour ne pas dépasser la limite imposée. Ceci implique souvent qu'il faut remanier le morceau de phrase choisi initialement. En effet, il se peut que la longueur de la phrase soit acceptable pour une langue comme l'anglais qui est assez concis et où les accents sont pour ainsi dire inexistants, mais tel n'est plus le cas pour l'allemand, dont les mots sont plus longs, ou le français, qui est truffé d'accents.

Au stade de la perforation apparaît également une autre servitude, car les terminologues dépendent alors entièrement du bon vouloir des perforatrices. Il est extrêmement rare que les personnes appelées à faire ce travail soient en même temps linguistes. Celles qui ont la maîtrise de toutes les langues, se consacrent sûrement à d'autres tâches mieux rémunérées.

$\mathrm{Si}$, par exemple, elles oubliaient de séparer les mots par un blanc, les résultats du tri automatique seraient désastreux. Même si dans ce cas la possibilité existe d'introduire une instruction supplémentaire en vue de la rectification automatique de cette erreur, il ne s'agirait là que d'un pis-aller valable pour une seule opération. Ceci implique donc une charge supplémentaire pour les linguistes qui eux doivent relire une liste off-line comprenant les textes de toutes les cartes perforées dans l'ordre numérique.

Une fois toutes ces données entrées en mémoire, le programme prévoit l'impression de listes où chaque mot est reproduit en face de la phrase qui l'entoure qui, à son tour, est suivie des phrases concordantes dans les autres langues. L'inversion des langues se faisant automatiquement, on disposera alors de cinq volumes distincts. Afin d'éviter la répétition des mêmes phrases et de réduire le volume des listes, une liste de mots noirs ou de mots vides a été dressée pour chacune des langues. Ces mots sont automatiquement éliminés par l'ordinateur en tant que mots clés. Initialement, la publication des glossaires s'est faite en cinq volumes. En vue d'en rendre la consultation plus facile, cette publication se fait maintenant en un seul volume comportant un corpus de phrases, dans l'ordre de leur numéro, assorti d'un index alphabétique pour chacune des langues. Ces index comprennent la liste des mots clés avec en regard les locutions dans lesquelles ils figurent. En choisissant les mots clés, le terminologue devra veiller soigneusement à ce que les mêmes termes ne soient repris dans l'index que lorsqu'ils donnent lieu à une variation de traduction dans une des autres langues. Les locutions qui figurent dans ces listes unilingues permettront au traducteur d'éliminer celles qui ne présenteront pas une analogie suffisante avec le texte qu'il doit tráduire.

Qu'il me soit permis d'ouvrir une parenthèse au sujet des textes possédant une valeur juridique. Si j'ai affirmé que l'analyse terminologique ne devrait se faire qu'à partir de textes originaux, ceci est vrai pour les textes scientifiques et techniques. S'il s'agit de textes qui possèdent une valeur juridique et dont on sait que les traductions dans d'autres langues ont la même valeur juridique, il faut non seulement analyser les originaux mais aussi les traductions. 
Tout comme dans les États fédéraux qui ont adopté un régime bilingue ou multilingue, les décisions et règlements de la Commission des communautés européennes sont publiés au journal officiel en quatre langues. Ces textes font foi dans chacune de ces langues et le traducteur doit donc pouvoir retrouver et reproduire le contenu exact de ces textes. Cependant, afin d'éviter que les traductions de moindre qualité se répètent ad eternam, il est prévu d'indiquer par un signe les autres traductions possibles lorsqu'il ne s'agit pas de citations textuelles.

La problématique juridico-linguistique telle qu'elle apparaît dans les nouvelles relations internationales et surtout sur le plan européen ${ }^{4}$ n'a pas encore été approfondie. Toutefois, comme j'ai eu l'occasion de le souligner lors de mon exposé sur la méthodologie de la recherche terminologique à l'occasion de la Conférence des doyens des facultés de droit, organisée par le Conseil de l'Europe il y a deux ans, la plus grande difficulté réside dans le fait que les mêmes termes ne couvrent pas les mêmes notions juridiques d'une langue à une autre ${ }^{5}$. Non seulement faut-il savoir que le terme français « décision » ne correspond pas toujours au terme anglais decision, encore faut-il être certain des différentes nuances et de la façon de les rendre. La moindre erreur de traduction pouvant entraîner un recours devant la Cour de justice des Communautés européennes à Luxembourg, la prudence et la précision s'imposent plus qu'ailleurs dans le domaine de la traduction des textes juridiques.

\section{CONSULTATION AUTOMATIQUE DIRECTE}

Un système qui ne vise que la publication de glossaires pour des domaines spécialisés ne présenterait qu'une solution partielle. Au fur et à mesure de la parution de ces ouvrages, le traducteur se trouvera en face de difficultés croissantes pour retrouver le volume où il pourra éventuellement trouver le ou les termes dont il a besoin. Les glossaires publiés à l'aide de l'ordinateur risquent comme tout autre ouvrage imprimé d'être périmés peu de temps après la date de leur parution. L'utilisateur devra donc à nouveau se consacrer à des recherches longues et difficiles.

La consultation automatique directe de la mémoire permet de poser des questions libellées dans une des langues retenues, en indiquant la langue cible dans laquelle le demandeur désire recevoir la réponse. Afin de rendre cette opération possible, il faut que l'ordinateur dispose de certains éléments de la structure de la langue qui lui sont fournis à l'aide de l'analyse morphologique de chacune des langues de départ. Grâce à cette analyse, qui est faite une fois pour toutes, la machine sera capable de reconnaître les mots sous toutes les formes qu'ils peuvent adopter dans un contexte. Le dictionnaire machine ainsi élaboré comprendra, par exemple, les radicaux et les désinences. Étant donné que les phrases introduites en mémoire ne contiennent pas uniquement des mots simples, mais également un grand nombre d'unités lexicales qui sont plus longues, telle «plaque de façade», il faut faire au préalable un certain travail de codage.

4. Georges Goedert, "Juristes, linguistes et Communautés européennes ", le Monde, 29 juillet 1969.

5. J. A. Bachrach, "La méthodologie de la recherche terminologique ", Conférence des doyens des facultés de droit organisée par le Conseil de l'Europe, Strasbourg, novembre 1968. 
Ce codage se fait pour tous les mots clés des phrases stockées. Le codeur devra tenir compte de l'ensemble des règles des manuels qui ont été élaborés à cet effet, ce qui constitue une autre servitude non négligeable, surtout lorsqu'il s'agit d'un système qui comprend plus de deux langues. Un des problèmes qu'il doit résoudre est de choisir entre la création de rubriques indépendantes pour chaque mot, la création de groupes locutionnels ou de rubriques d'homographes pour enregistrer les différentes possibilités de traduction. Toutes les entrées du dictionnaire sont assorties du numéro de la phrase stockée et du sigle du code-matière auquel elle appartient ${ }^{6}$.

L'utilisateur du système marquera dans le texte qu'il doit traduire tous les mots qui présentent pour lui une difficulté de traduction. Une photocopie du document ainsi marqué est envoyée au bureau de terminologie, qui introduit les termes entourés de la partie du contexte qui s'y rapporte directement dans la machine en indiquant la langue cible dans laquelle la traduction est demandée. Grâce aux données dont l'ordinateur dispose à la suite des analyses morphologiques que je viens de mentionner, on n'a nullement besoin de remanier le texte pour ramener par exemple les verbes à leurs infinitifs ou les pluriels des substantifs au singulier. L'introduction de la question peut avoir lieu au moyen de la perforation de cartes ou bien moyennant la console lorsque le système est intégré dans un circuit de traitement à distance.

D'autres systèmes obligent le terminologue à ramener tous les mots contenus dans la demande à leurs formes canoniques respectives avant d'introduire les questions dans la machine. Les réponses de l'ordinateur se présenteront alors sous forme de listes de mots en ordre chronologique, qui contiennent toutes les informations stockées concernant ces termes pour un couple de langues donné. Toutefois, l'avantage d'une présentation des mots dans leur contexte et de l'analyse morphologique des langues au préalable est que la machine fait un tri sélectif et élimine les redondances d'informations dont le client n'a nullement besoin.

Le programme prévoit la consultation de tous les fichiers qui se trouvent en mémoire machine pour que l'ordinateur puisse comparer le texte de la question avec toutes les données stockées. À la suite d'un calcul du plus grand nombre d'analogies, il choisira la ou les phrases qui correspondent le plus à la phrasequestion.

Le résultat d'une telle consultation prendra la forme d'un mini-glossaire bilingue où les mots clés avec leurs contextes ne sont pas présentés en ordre alphabétique, mais dans l'ordre de leur apparition dans le texte à traduire. Ce mini-glossaire étant produit en deux exemplaires, les terminologues peuvent facilement contrôler le contenu pour combler les lacunes indiquées par la machine. Qu'il me soit permis de donner quelques exemples puisés dans ce genre de listes (cf. Annexe).

\section{CONCLUSION}

Cela prouve que la machine est un serviteur exemplaire, puisqu'elle fait elle-même connaître ses défaillances. Mais, ses défaillances, comme ses prouesses, sont néanmoins le fait de l'homme. La machine est maître dans la mesure où elle

6. J. A. Bachrach et L. Hirschberg, "Une troisième version du DICAUTOM ", 2 Conférence internationale sur le traitement automatique des langues, Grenoble, 23-25 août 1967. 
impose à l'homme une certaine rigueur et une certaine discipline et le contraint à adapter ces méthodes de pensée et de travail. Cependant, rigueur et discipline sont toujours indispensables car, pour citer l'auteur belge Henri de Man ${ }^{7}$ : «la discipline est comme les rives d'une rivière qui sans cela s'étend en marécage ». De même, le contexte canalise notre pensée et donne leur signification aux mots. Dans cet ordre d'idées, on pourrait considérer que l'ordinateur fait fonction de moulin à eau : il reçoit la masse verbale, la traite et la transforme en un produit consommable.

Actuellement, lorsqu'on pense mots et machine, instinctivement on arrive à associer les deux en banque de mots. Jusqu'ici, les seules tentatives connues sous cette dénomination semblent avoir comme objectif la sauvegarde d'une langue donnée, à savoir le français. Est-ce parce que les banques d'information dont parle Louis Armand dans son livre le Pari européen ne donnent que des renseignements en une langue, que les banques de mots ne cherchaient jusqu'à ce jour qu'à amasser des trésors unilingues. Je suis sûr que tel ne sera point le cas de la banque de mots qui a été inaugurée au cours de ce congrès.

Le monde que nous connaissons dispose de moyens de communication, certes non sans faille, mais ultra-rapides. En principe, les messages peuvent être transmis et reçus pratiquement à l'instant, encore faut-il qu'ils soient compris. De même pour les communications directes entre hommes qui peuvent facilement se réunir autour d'une table mais qui doivent, cependant, se comprendre pour aboutir. La pluralité des langues, outils majeurs pour exprimer nos pensées, a dressé des barrières linquistiques qui ne peuvent être surmontées qu'à l'aide de la traduction.

Qu'on ne vienne pas me dire que les experts se comprennent toujours, car comment se fait-il alors que même pour ceux qui emploient une langue commune, les mots dont ils usent prennent si souvent des sens très différents ? Qu'il me soit permis d'en donner un exemple qui démontrera l'avantage d'une discipline rigoureuse qui exige que l'on enregistre soigneusement les références bibliographiques.

Après la publication d'un de nos glossaires, une traductrice anglaise nous a fait remarquer qu'elle ne pouvait accepter l'emploi du terme broadcaster dans les deux contextes que nous proposions. Sans ces contextes, on n'aurait pas tout de suite compris de quoi il s'agit. Car même l'indication « agriculture» n'aurait pas suffi. Le Webster propose comme définition : «a mechanical device for sowing seed, especially of grass or clover, by scattering it broadcast over the ground, usually by centrifugal force ». Voyons voir les contextes donnés :

AG 024250

F. la puissance nécessaire de la prise de force doit être plus grande pour un distributeur d'engrais centrifuge que pour un distributeur en nappe

E. for a broadcaster with large spreading width more power take-off capacity is required than for a machine with small spreading width (fertilizer broadcaster)

Réf. : f/CNEE II 81, e/BSI 13-50

$A G 024293$

F. on peut fixer simultanément aux tracteurs porte-outils des distributeurs d'engrais, des semoirs et des planteuses automatiques de pommes de terre

E. simultaneous utilization of a fertilizer broadcaster and a seed drill or automatic potato planter behind a self-propelled tool carrier is possible

Réf. : e/BSI 13-50

7. Henri de Man, Cahiers de ma montagne. 
Sur notre demande, cette correspondante nous a déclaré avoir consulté le British Standard Glossary of Terms Relating to Agricultural Machinery and Implements (BS 2468 : 1963) et en être arrivée à la conclusion que broadcaster est le terme exact dans le cas du distributeur d'engrais mais qu'il vaut mieux parler de spreader dans le cas où le distributeur d'engrais couvre également la notion d'épandeur de fumier de ferme. Nous avons pu lui faire connaître notre source, également une publication du British Standards Institute qui comprend une description du broadcaster. Enfin, nous nous sommes mis d'accord pour accepter une nouvelle version pour la première locution étant donné que le terme français engrais, tout en faisant penser d'abord aux engrais chimiques, englobe néanmoins le fumier également (4250 nouvelle version : « a fertilizer broadcaster with a wide spread needs a higher p.t.o. (power take-off) than a manure spreader $\gg$ ).

C'est ce genre d'information qui est utile et qui forme ce que l'on appelle en informatique le feed-back ou rétroaction. Étant donné que le numéro de la phrase et la rubrique du code-matière sont connus, il est très facile de faire enlever les données erronées et de les remplacer dans les fichiers.

À défaut de contexte, le terme broadcaster nous ferait d'ailleurs penser en premier lieu à l'annonceur ou présentateur à la radio ou à la télévision. Par analogie, on pense à «speaker», terme anglais mais figurant au Robert, car André Gide lui-même semble l'avoir employé. Des speakers aux speakerines, ces charmantes personnes qui présentent avec un aimable sourire les programmes de la télévision, il n'y a qu'un pas. Peut-être pourrais-je vous proposer dans le contexte du dictionnaire automatique et des banques de mots : diffuseur et notamment diffuseur des connaissances terminologiques. Ce terme également se trouve dans le Robert ${ }^{8}$, et nous conviendrait bien. Car, ne s'agit-il pas en fin de compte, d'un dialogue hommemachine, où l'homme rassemble les données pour les emmagasiner dans la mémoire électronique, tandis que la machine, elle, fait le tri plus rapidement que l'homme et répond par un courant d'informations sélectionnées aux questions que lui pose l'homme. Dans ce courant d'informations, le mot acquiert son vrai sens et permettra à l'homme de mieux rendre les pensées exprimées dans une langue qui n'est pas comprise par tout le monde. Les mini-glossaires phraséologiques dont je vous ai parlé donnent au traducteur la possibilité de trouver le mot juste en comparant les termes figurant dans les phrases-exemples avec les termes et tournures de phrases du texte qu'il est appelé à traduire. Ainsi il gagnera un temps précieux qui viendra à point pour améliorer le style de sa traduction en s'inspirant des locutions que lui propose la banque de mots.

MacLuhan a raison lorsqu'il arrive à la conclusion que les moyens de communication ultra-rapides dont dispose l'homme ont ramené le monde à l'échelle du village en opérant un rapprochement entre peuples et continents. L'homme seul ne peut plus aboutir dans ces recherches sans contacts avec d'autres pour obtenir l'information souhaitée.

Ainsi, les initiateurs de systèmes de renseignements terminologiques rapides doivent se mettre en rapport avec le plus grand nombre d'organismes actifs dans ce domaine où qu'ils se trouvent dans le monde. Le réseau de renseignements qui a été créé à Luxembourg comprend évidemment des institutions canadiennes, tels

8. Paul Robert, Dictionnaire alphabétique et analogique de la langue française. 
le Secrétariat d'État, les publications de l'Université de Montréal et autres, RadioCanada, les chemins de fer et j'en oublie certainement, avec qui l'échange de publications constitue une collaboration des plus fructueuses.

Les informations empruntent d'ailleurs souvent des chemins tortueux pour arriver à destination. Ainsi, il fallait qu'à Luxembourg nous consultions l'excellent bulletin mensuel publié par le Centre de terminologie du Bureau des traductions du Secrétariat d'État à Ottawa, pour apprendre qu'à la suite d'une vaste enquête le Comité d'étude des termes techniques français, en collaboration avec l'A.F.I.R.O. (Association française d'informatique et de recherche opérationnelle) a retenu le terme périgramme pour rendre le terme anglais software. Depuis, une intervention de M. Maillot, après l'exposé de M. Geleff, m'a appris qu'il ne faut plus employer "périgramme » mais que l'AFNOR a décidé de remplacer ce terme par «programmerie ». Je ne pense pas que ce soit ici l'occasion rêvée d'entamer une discussion sur le bien-fondé de ce changement. Je désire seulement souligner que c'est encore grâce à une initiative canadienne qui m'a valu d'être ici que nous saurons à Luxembourg qu'il faudra apporter d'urgence un changement à notre trésor terminologique.

Malgré les distances et la vaste nappe d'eau qui nous séparent, la terminologie se propage de foyer à foyer comme dans un village où tout le monde se connaît et où le banquier fait confiance au client pour que celui-ci lui confie les résultats de son travail pour le faire fructifier dans le trésor commun.

J. Albert Bachrach

ANNEXE

Exemples de deux extraits de mini-glossaires, obtenus par suite d'une consultation directe de la machine

$$
\text { Français - Anglais }
$$

jetée pour les chargements de pétroliers

jetée d'accès avec plate-forme pour chargement et déchargement de pétroliers pier with platform for loading and unloading of tankers

caractéristiques physiques des aciers de construction

évolution des caractéristiques de l'acier destiné à la construction métallique improvements in the properties of steel used in building and construction caractéristiques physiques ou mécaniques physical or mechanical properties

TRB2

chers en béton travaillant au cisaillement

chape flottante en béton

floating concrete layer

résistance du cisaillement de planchers en béton préfabriqué

shear resistance of precast concrete floors

longueur réelle des membrures tubulaires travaillant à la compression

effective length of tubular compression members

INA5

INH5

pose des panneaux de façade sur la charpente

pose des panneaux de remplissage de la charpente

fixing of wall cladding panels on steel frame

panneaux de façade courants ordinary front panels

INO5

INC5

INA5

lages par boulons à haute résistance ou par soudure soudure par résistance

resistance welding

assemblage par soudure ou par brides mobiles

connection by welding or by lose flanges

le soudage et l'emploi de boulons à haute résistance se substituent au rivetage welding and the use of high-strength bolts replace riveting

INA5

INO5

INC5

IND5

INB5 


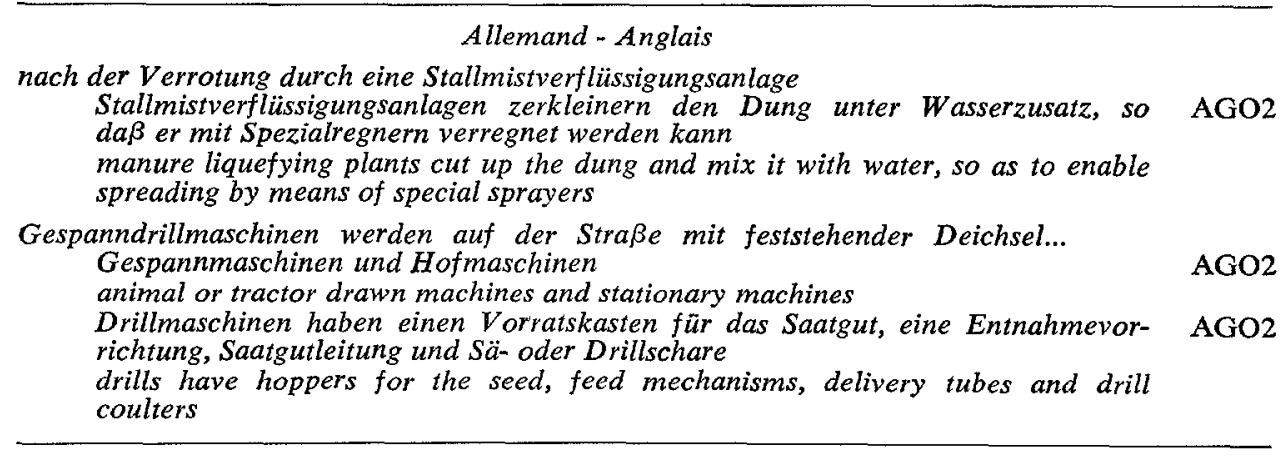

\section{QUESTIONS}

M. Maillot : Le thème de cette journée du colloque, c'est l'ordinateur (banque de mots) et le traducteur. Lorsqu'un traducteur s'installe à son compte, il est obligé de faire certains investissements, d'acheter de la documentation, des dictionnaires, de s'abonner à des revues. Je ne sais pas, n'ayant pas l'expérience de ce statut de traducteur, à combien cela peut se monter? La création d'une banque de mots entraîne certainement des investissements considérables et je me demande comment doit-on voir la question pour un traducteur appelé à consulter la banque de mots? Devra-t-il souscrire un abonnement? Doit-on comprendre que les organismes, surtout sur la plan national, qui s'occuperont de ces banques de mots considéreront que les services qu'elles rendent au traducteur sont entièrement gratuits ?

Réponse : J'ai parlé d'une réalisation à l'intérieur d'une seule organisation pour l'instant, donc là, forcément nous sommes surtout au service de nos traducteurs, mais nous recevons souvent des demandes de l'extérieur, de freelance que nous employons parfois. Ils ont droit aux renseignements et ce n'est pas par altruisme, c'est très égoïste même. Nous sommes heureux d'accueillir des demandes de l'extérieur pour cette simple raison que cela nous apporte quelque chose, l'idée d'un domaine où l'on n'a encore rien, surtout quand, de différents côtés, on nous demande la même chose, ou du moins des renseignements qui ont trait à un même secteur d'activités. Au début, on est très content de donner les renseignements gratuitement. Je suppose qu'on devrait peut-être adopter la formule des abonnements. $M$. Geleff : Vous nous avez dit que vous partiez toujours d'un texte original du mot pris dans son contexte original et ceci m'amène à vous poser deux questions. Lorsque vous avez élaboré votre méthodologie est-ce que vous avez pris cette décision à priori, ou bien si vous l'avez prise à cause d'une expérience que vous auriez pu acquérir en cours de travail ? Lorsque vous travaillez sur un texte original, dans quelle mesure pouvez-vous être certain que l'auteur n'a pas été influencé par des vocabulaires étrangers, par des traductions dont il a eu connaissance, à droite ou à gauche ?

Réponse : Créer une banque de mots, c'est un travail d'équipe. Dieu sait qu'on a besoin de l'aide de tout le monde ! Justement toutes les expériences dont on prend connaissance dans le monde entier nous aident. Une très grande bêtise serait de vouloir créer quelque chose et de ne jamais se baser sur les recherches et les expériences qui ont déjà été faites ailleurs. Ceci, c'est seulement un préalable pour vous dire que c'était d'après mon expérience personnelle. J'ai été longtemps traducteur au service d'organisations internationales, j'ai donc pu voir en révisant des textes combien il est facile de laisser échapper des fautes, parce que les traducteurs et les réviseurs sont toujours extrêmement pressés. Quels sont les critères sur lesquels nous nous basons pour évaluer un peu la qualité du texte original ? Le jugement humain de toute façon est toujours subjectif, son objectivité, en tout cas, est très relative. Nous nous basons sur les indications de source des textes originaux. Nous nous adressons à beaucoup de centres de documentation et de recherche dans les différents pays membres et même des nonmembres des communautés européennes qui travaillent dans le secteur où nous devons 
faire des analyses terminologiques pour l'établissement de nos glossaires et pour l'augmentation de notre trésor. C'est déjà une certaine référence, je suppose que ces organismes-là, par exemple l'AFNOR, ne vont certainement pas nous indiquer des normes dans un langage qui n'est pas sain. Quand il s'agit d'articles publiés, il y a le sérieux de la revue, de l'éditeur de la revue, le renom de l'auteur, et nous faisons encore un tri dans toute cette masse documentaire en regardant soigneusement le contenu. Je crois qu'on ne peut pas vraiment normaliser ce genre de critères; il faut avoir un peu de jugeotte.

M. Darbelnet : Au cours de votre exposé vous avez soulevé un point sur lequel j'aimerais avoir une confirmation. Si jai bien compris, vous n'êtes pas en faveur d'une insertion brutale des dictionnaires existants dans l'or- dinateur, vous les soumettriez à un contrôle. Réponse : Certainement à un contrôle. Il faut voir d'après quels principes ils ont été élaborés, parce qu'il y a une série de dictionnaires qui ne servent à rien, au contraire, ils peuvent induire le traducteur en erreur. Vous devez toujours réorganiser parce qu'il serait ridicule de simplement copier un dictionnaire parce que la machine peut faire le tri que vous faites en le feuilletant. Nous préférons travailler par petits secteurs, faire l'analyse nous-mêmes, vérifier si nous trouvons quelque chose de bon dans certains dictionnaires et prendre l'information. De toute façon, il n'y a pas de droits d'auteur parce que la banque de mots se doit d'être honnête et divulguer la source, pour souligner le sérieux de son trayail, le sérieux de son information. 